\title{
GESTÃO DAS TAREFAS EM PROCESSOS INDUSTRIAIS
}

\section{TASKS MANAGEMENT AT INDUSTRIAL PROCESSES}

\author{
Elísio Carvalho Silva ${ }^{1}$ \\ ${ }^{1}$ Universidade Federal da Bahia - UFBA - Bahia - Brasil \\ elisio.carvalho@terra.com.br
}

\begin{abstract}
Resumo
Os erros humanos são fatores contribuintes para ocorrência de grandes acidentes nas indústrias químicas, petroquímicas, óleo e gás. Isso ocorre porque os processos estão cada vez mais automatizados em função do avanço da tecnologia e das grandes demandas de consumo. Esse fenômeno contribui para aumentar a possibilidade dos erros humanos, visto que os operadores reduzem a sua atuação, passando mais para um processo supervisório e perde a sua habilidade para intervir no processo. Daí surge a importância de uma atenção especial nas tarefas. As tarefas críticas têm papel fundamental na composição das causas de acidentes, por isso é essencial ser feita uma boa gestão para reduzir a possibilidade de erros humanos. Este artigo busca uma discussão dos erros humanos ao destacar várias opiniões de autores e, por fim, apresenta uma proposta para gestão das tarefas que inclui desde a identificação daquelas críticas, análise dos passos da tarefa, elaboração do procedimento, treinamento, simulados e avaliação de oportunidade de melhoria. $O$ resultado final é a redução da probabilidade de erros humanos em tarefas consideradas críticas e consequentemente a redução de acidentes no setor industrial.
\end{abstract}

Palavras-chave: tarefas; gestão; acidentes; erros humanos.

\section{Introdução}

Nas indústrias química, petroquímica, óleo e gás as tarefas realizadas nos processos de produção normalmente envolvem situações de risco. Por isso, há uma necessidade de gerenciamento adequado para minimizar a possibilidade de erro humano e, assim, reduzir a frequência de eventos indesejados. Muitos acidentes já ocorreram devido a falhas em procedimento: ou por ele está mal elaborado, ou por falha humana em segui-lo. O acidente da usina nuclear de Three Mile Island provocou bastante repercussão mundial, porque foi o primeiro grande acidente já ocorrido em plantas de geração de vapor deste tipo. Um dos fatores contribuintes desse acidente foi a indisponibilidade do sistema auxiliar de emergência de água de refrigeração, visto que havia duas válvulas fechadas quando a planta retornou após dois dias de manutenção (PERROW, 1984).

Para reduzir a probabilidade de erro, é preciso que as tarefas sejam analisadas e verificadas os potenciais de erro humano e daí determinar ações para minimizar essas possibilidades. Uma ferramenta bastante útil para tornar os processos industriais mais seguros é a análise de tarefa. A 
análise de tarefa é uma metodologia que além de definir o treinamento, identificar os riscos envolvidos em cada passo da tarefa, controles, etc., também é útil para determinar quantas pessoas serão necessárias para efetuá-la. Swain e Guttmann (1983) destacam que antes do acidente de Three Mile Island as organizações não utilizavam essa metodologia para esse fim.

Com o aumento da tecnologia, os processos passam, cada vez mais, a ser automatizados e possuir um nível elevado de redundância que contribui para eles se tornarem mais seguros. Conforme a API 770 (2001), essas redundâncias minimizam as consequências de falhas de componentes. Contudo, os erros humanos aumentam e causam cerca de $90 \%$ das probabilidades de falhas no sistema, principalmente aquelas relacionadas às falhas sistêmicas. Essas falhas têm as seguintes origens: na avaliação de risco; no projeto; manutenção; especificação; instalação; comissionamento; gerenciamento de mudança, etc. Por tudo isso, é fundamental que empresas do segmento industrial tenham uma boa gestão da tarefa. Dessa forma, haverá redução da possibilidade de falha humana e consequentemente redução nos acidentes o que será valioso para as indústrias e a sociedade. Este artigo tem como objetivo propor um programa de gestão para que uma tarefa seja realizada com a menor possibilidade de erro humano. Espera-se que contribua no melhoramento das gestões de tarefas nesse segmento industrial.

\section{Erro humano}

Leveson (2011) define o erro humano não intencional em duas categorias: deslizes (slips) e enganos (mistakes). Os deslizes são erros que surgem de uma intenção correta, porém o executante faz a ação errada. Por outro lado, os enganos são erros que ocorrem no planejamento de uma ação, ou seja, partem já de uma intenção errada. Por exemplo, no caso do deslize, o operador decide parar a bomba A, no entanto, ao tomar a ação ele para a bomba B. Já no engano, o operador decide parar a bomba B (parte do planejado), porém seria a bomba A que deveria ser parada.

A HSE (2005) e CCPS (1994) também definem o erro não intencional como deslize e engano. Essas organizações adicionam que os deslizes ocorrem na execução de tarefas familiares, tais como, esquecer-se de fazer algo relevante durante uma manutenção, calibração ou teste. Já os enganos surgem quando o comportamento é baseado em relembrar regras, ou procedimentos familiares, ou situações não familiares onde é preciso tomar decisões baseadas em conhecimento e julgamentos. Reason (1990) adiciona os lapsos como uma variante dos deslizes. Esses tipos de erros podem passar despercebidos pela própria pessoa que o cometeu, pois é uma falha de memória.

Adicionalmente, existem os erros intencionais os quais são também importantes no contexto do erro humano. Embora sejam realizados conforme a vontade própria das pessoas, eles são raramente feito propositadamente (HSE, 2005), exceto a sabotagem. Esses erros estão relacionados ao não cumprimento de procedimento, atalhos inapropriados, etc. A intenção da pessoa é fazer com 
que o trabalho seja realizado, independente das consequências, uma vez que não acredita no que está escrito, ou deseja acelerar algum processo para obter feedback de reconhecimento. As causas que levam a essas situações são diversas: procedimentos não são revisados conforme as mudanças ocorridas e isso os deixam desacreditados; falta de envolvimento das pessoas na elaboração do procedimento e, então, elas acham que existe outra forma melhor de efetuar a tarefa; procedimentos muito extensos e complexos que os tornam difíceis de executá-los; falta de disciplina operacional (API 770, 2001; CCPS, 1994; BELL; SWAIN, 1983; SILVA, 2009).

Os erros também podem ser denominados de omissão (omission) ou de execução (comission). Os erros de omissão são aqueles em que o operador esquece-se de fazer algo (lapsos), ou deliberadamente decide não fazer (violações). Já os erros de execução, o operador erra no momento da execução da tarefa (deslizes/lapsos ou enganos) ou propositadamente faz diferente do que deveria ser feito (violação).

\section{Erro humano em sistemas complexos}

Os sistemas complexos favorecem a ocorrência do erro humano. Leveson (2011) diz que em sistemas altamente automatizados o operador fica dependente deles. Isso leva a um novo tipo de erro, que é a confusão dos modos de falha do sistema automatizado o que aumenta os erros de omissão e execução. Complementando, Mannan (2005) ressalta que à proporção que os sistemas se automatizam, a atividade de controle do operador se reduz, em contrapartida aumenta a de monitoramento. Essa mudança reduz a intervenção humana com o sistema e ao longo do tempo o ser humano reduz a sua capacidade de resposta. CCPS (1994) adiciona que a habilidade do operador será deteriorada com o aumento da automação. A causa disso é porque o operador deixa de praticar e, assim, perde a experiência previamente obtida.

Perrow (1984) menciona que os acidentes são inevitáveis devido a interatividade complexa entre os sistemas e também quando eles são fortemente acoplados. Dessa maneira beneficia o surgimento de causas comuns de falhas as quais são provenientes de eventos randômicos ou sistêmicos. No entanto, destaca que é possível reduzir a possibilidade de acidente por essas causas com melhores projetos, equipamentos e procedimentos. Exemplo recente foi o acidente do poço de petróleo Macondo, no Golfo do México, em 2010, onde o sistema de prevenção de explosão (blowout preventer) falhou e os sistemas auxiliares de prevenção também falharam (MARINE BOARD, 2011). Nessa mesma linha, Leveson (2011) menciona um acidente ocorrido num reator de batelada, na Inglaterra, onde a lógica do sistema básico de controle de processo estava errada (falha sistêmica) e não permitiu controlar a temperatura do reator.

À proporção que os sistemas ficam complexos, é necessário fornecer mais ferramentas para o operador desempenhar a sua tarefa, pois ele necessita aumentar a sua habilidade cognitiva. Quanto 
maior a complexidade, maior será a probabilidade de erro humano. Bridges (2011) considera que o erro humano pode aumentar de 2 a 10 vezes em função da complexidade. Na sua visão, a complexidade de uma tarefa é proporcional ao número de escolhas disponíveis para fazer uma seleção errada (equipamentos semelhantes), número de tarefas paralelas que pode distrair o operador da tarefa principal, número de pessoas envolvidas, julgamento e cálculos necessários. Bird, Germain e Clark (2007) chamam essas tarefas como críticas e as relacionam com a severidade de causar danos, frequência de exposição às pessoas e probabilidade da tarefa dar errada. A probabilidade é função da periculosidade, dificuldade, complexidade e a chance de ter uma perda se ela for feita de maneira inapropriada.

Swain e Guttmann (1983) vão além dos sistemas complexos. Eles chamam atenção para os fatores moduladores de desempenho (PSF), internos e externos, os quais influenciam a probabilidade de erro humano. Os fatores internos estão relacionados com as características individuais da pessoa, estressores e estereótipo populacional. Enquanto que os fatores externos são influenciados pelo ambiente do trabalho, principalmente o projeto do equipamento e procedimentos escrito ou instrução oral. Outra contribuição é a proveniente do CCPS (1994) que chama fatores semelhantes ao PSF como fatores que influenciam o desempenho humano (PIF). Eles estão basicamente calcados em procedimentos, ambiente físico do trabalho e fatores sociais. Os fatores sociais são os internos a empresa e estão intimamente ligados a distribuição da carga de trabalho, clareza das responsabilidades, comunicação, estrutura do grupo e da liderança e planejamento do grupo e orientação. No mesmo caminho estão Ávila, Pessoa e Andrade (2013), que propõem uma nova ferramenta chamada de Hazop social para efetuar as análises desses fatores modeladores de desempenho.

\section{Mecanismo de erro, tarefa e intervenção humana}

Uma tarefa é um segmento do trabalho no qual necessita de um conjunto de ações específicas e distintas para a sua conclusão. O conjunto de ações será realizado pelo ser humano, portanto um erro humano poderá causar consequências na produtividade, na qualidade, na segurança ou no meio ambiente.

Os mecanismos de erro do operador estão definidos em três níveis de comportamento, conforme menciona Rasmussen (1982/1983): comportamentos baseados na habilidade, regras e conhecimento. No mecanismo de comportamento baseado em conhecimento, o operador faz uma leitura do problema, os diagnósticos necessários e elabora um plano de procedimento para a solução do problema; são utilizados conhecimentos. No comportamento baseado em regra o operador ver o problema e utiliza procedimento já preestabelecido. Já no comportamento baseado na habilidade ele 
olha a situação e busca rotinas já armazenadas na mente e toma ação. Nesse caso, são ações já bastante praticadas anteriormente e as faz automaticamente.

Reason (1990) relaciona os tipos de erros com os comportamentos de erros no que ele chama de sistema genérico de modelo de erro (GEMS). Portanto, os tipos de erros humanos podem ser associados aos mecanismos de erros nos seus três níveis de comportamento. Os erros chamados deslizes/lapsos estão intimamente relacionados com o comportamento baseado na habilidade, onde o operador, por exemplo, se confunde ao alinhar determinado equipamento ou uma válvula. Ele sabe o que fazer, mas devido a problema de layout, falta ou falha de identificação causa esse tipo de erro. Contudo, há uma grande possibilidade dele recuperar o erro e evitar que algum incidente de processo ocorra, desde quando o tempo necessário para ocorrer a consequência seja suficiente para permitir a recuperação do erro.

Os enganos estão relacionados tanto com o comportamento baseado em regras como o baseado em conhecimento. Quando é feita uma operação de rotina (baseada na habilidade) e o resultado não é o esperado, o operador passará para o comportamento baseado em regras, conforme mostra Figura 1. Verificará quais os procedimentos e informações terá que se lembrar para diagnosticar o problema e solucioná-lo. No entanto, essa última ação poderá não ser suficiente, e o próximo passo será utilizar todo o seu conhecimento e ser suficientemente resiliente para encontrar a solução para o problema. A resiliência é necessária porque, possivelmente, nem todas as ações estarão escritas em procedimento ou o procedimento poderá estar errado (HOLLNAGEL; WOODS; LEVESON, 2008). Nesse momento já é o inicio de uma resposta a uma possível emergência. O evento indesejado ocorrerá se o operador não tiver conhecimento dos procedimentos, o procedimento estiver incorreto ou não dominar profundamente o processo operacional por meio de treinamento e simulações. A falta desses suportes levará a interpretações erradas das variáveis de processo culminando com diagnóstico e ação falsos. A Tabela 1 mostra o relacionamento entre comportamentos e tipos de erro. 
Figura 1- Fases da solução de problemas

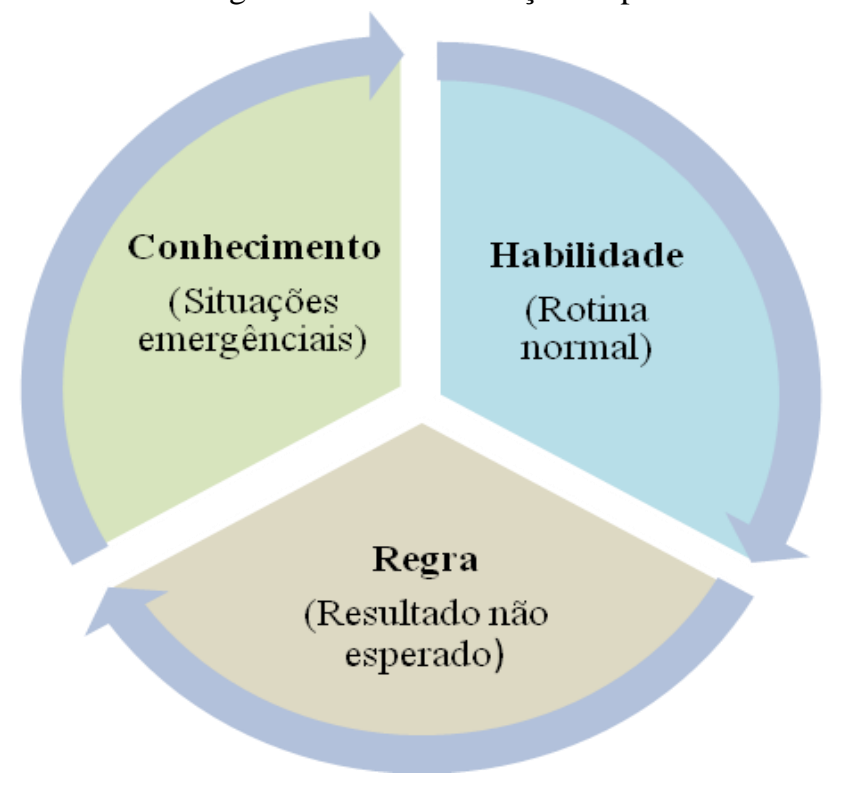

Fonte: Adaptado de Reason (1990)

Tabela 1 - Comportamento e tipos de erro

\begin{tabular}{|c|c|c|c|}
\hline \multirow{5}{*}{$\begin{array}{l}\text { Comportamento e tipos } \\
\text { de erros }\end{array}$} & $\begin{array}{c}\text { Baseado na } \\
\text { habilidade } \\
\text { Deslizes/lapsos }\end{array}$ & $\begin{array}{c}\text { Ações baseadas em } \\
\text { conhecimentos passados que } \\
\text { se tornaram rotinas. São } \\
\text { realizadas com baixo nível } \\
\text { de cognição }\end{array}$ & $\begin{array}{l}\text { Baixo nível de } \\
\text { cognição - ações } \\
\text { automáticas }\end{array}$ \\
\hline & $\begin{array}{c}\text { Baseado em } \\
\text { regras }\end{array}$ & \multirow{2}{*}{$\begin{array}{c}\text { Ações baseadas em } \\
\text { procedimentos ou outras } \\
\text { informações }\end{array}$} & \multirow{2}{*}{$\begin{array}{l}\text { Médio nível de } \\
\text { cognição - ações } \\
\text { transientes entre } \\
\text { automáticas e } \\
\text { conscientes }\end{array}$} \\
\hline & Enganos & & \\
\hline & $\begin{array}{c}\text { Baseado em } \\
\text { conhecimento }\end{array}$ & \multirow{2}{*}{$\begin{array}{l}\text { Ações baseadas no } \\
\text { conhecimento }\end{array}$} & \multirow{2}{*}{$\begin{array}{l}\text { Alto nível de cognição } \\
\text { - ações totalmente } \\
\text { conscientes }\end{array}$} \\
\hline & Enganos & & \\
\hline
\end{tabular}

Fonte: Adaptado de CCPS (1994) e Reason (1990)

Por tudo isso, Lidwell, Holden e Butler (2010) defendem que projetos devem ser concebidos para ajudar as pessoas a evitar erros e minimizar as consequências negativas dos erros caso eles ocorram. Um dos princípios a ser usado é o chamado forgiveness (perdão) que pode utilizar as seguintes características: a) affordances- que representam as características físicas do projeto que influenciam o seu uso correto, por exemplo, engates diferentes para produtos químicos incompatíveis; b) reversibilidade da ação - uma ou mais ações serão revertidas caso ocorra um erro. A utilização de check list pode ajudar na recuperação do erro se preenchido após a ação. Se preenchido antes, evitará o erro; c) confirmação - verificação da intenção antes da ação ser concretizada, por exemplo, ao executar o primeiro passo, fará a seguinte pergunta: “quer realmente fazer esta ação?”; d) avisos - sinais, identificações, alarmes para avisar sobre iminentes riscos. Um exemplo bastante utilizado no segmento industrial são alarmes de variáveis fora de controle; e) ajuda - informação para ajudar nas operações básicas e matriz de solução de problemas os quais podem ajudar na recuperação do erro. 


\section{Análise da tarefa}

Com a necessidade de cada vez mais produzir em alta escala e ser competitivo globalmente, tornou-se necessário aumentar a automação, assim como surgiram novas tecnologias para a melhoria dos controles do processo. Com isso, os grandes acidentes se tornaram menos frequentes. No entanto, quando eles ocorrem há uma grande quantidade de energia envolvida em função de grandes massas de produtos perigosos manuseados, o que leva a grandes perdas a incluir perdas humanas. Alguns exemplos mais recentes como Buncefield em 2005, British Petroleum em Texas City também em 2005, British Petroleum no Golfo do México em 2010, PDVSA em 2012 na refinaria de Amuay na Venezuela e Pemex em 2012 na cidade de Reynosa no México comprovam esses fatos. Esses fenômenos forneceram maior grau de complexidade aos processos, assim como mantiveram os operadores menos ativos com um papel mais supervisório que de controle e intervenção.

A automação tem o intuito de aumentar a segurança nos processos industriais. Nos cenários de acidentes mais críticos identificados em análise de risco são adicionadas camadas de proteção. Essas camadas, às vezes, são sistemas instrumentados de segurança (SIS), que tomarão ação imediata se o sistema básico de controle (outro sistema automático) falhar. Por ter essas proteções, o operador reduzirá a sua interferência quando o sistema tender a perder o controle, e isso induzirá a redução da sua habilidade em lidar em caso de uma resposta rápida se um SIS falhar. Embora controverso, também há a teoria do efeito da compensação ou "homeostase" do risco (GELLER, 2000). Presume-se que as pessoas ajustam sua percepção de risco quando percebe que um sistema é seguro. Ou seja, sendo mais seguro é possível chegar ao limite das camadas de proteção, tornando ainda mais factível a ocorrência do acidente. Adicionalmente, deve-se levar em consideração que algumas emergências ocorrem por falha na instrumentação, o que força ações do operador para colocar o processo nas condições normais. Caso ele não esteja preparado, o acidente ocorrerá. Um caso recente, que teve repercussão mundial, foi o acidente da Air France do voo AF 447. Nesse acidente, os dois tubos de pitot falharam por possível obstrução por cristais de gelo (causa comum de falha), e os pilotos não souberam lidar com o cenário (BEA, 2012).

Dadas essas circunstâncias, é preciso dar uma maior atenção ao ser humano para lidar com cenários mais críticos que possam perder o controle. Existem várias metodologias de análise da tarefa que podem identificar erros humanos. Entre elas estão o HAZOP da tarefa, What if da tarefa, análise hierárquica de tarefa, análise da tarefa, erro humano preditivo e análise de erro de ação (BRIDGES; WILLIAMS, 1997; MANNAN, 2005; EMBREY; ZAED, 2006, BIRD; GERMAIN; CLARK, 2007; SWAIN; GUTTMANN, 1983). Qualquer uma dessas metodologias fará análise passo a passo da tarefa para prever as situações de risco e fornecer orientações para redução da probabilidade do erro humano. A análise de tarefa também proporciona outros ganhos, tais como: 
fornece base para a necessidade do desempenho humano versus a sua capacidade; provê informações para desenvolvimento do procedimento; ajudam a identificar a quantidade de pessoas para executar a tarefa, necessidades de treinamento e comunicações (REED; SHAFFER, 2008; MANNAN, 2005).

\section{Gestão das tarefas}

Todas as tarefas devem ser gerenciadas, porém aquelas mais críticas devem possuir uma gestão mais robusta, porque as consequências de um erro podem causar ferimentos em pessoas ou danos à propriedade e ao ambiente. Neste artigo será proposta uma gestão para as tarefas, conforme mostra a Figura 2.

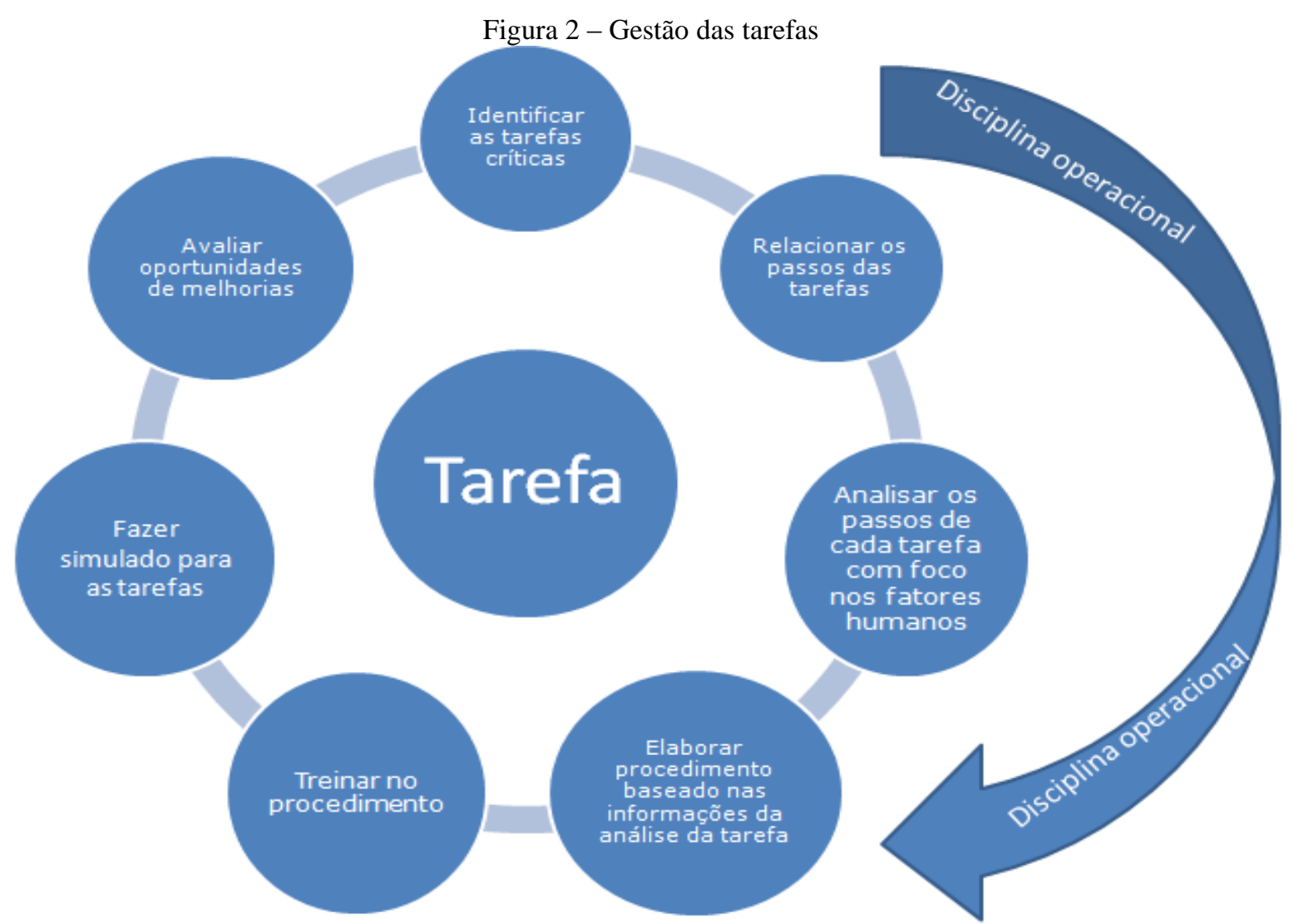

Fonte: Adaptado de Bird, Germain, Clark (2007) e Silva (2009)

\subsection{Identificar as tarefas críticas}

Normalmente as empresas já conhecem as suas tarefas. No entanto, é importante que nessa fase seja criado um grupo multidisciplinar para revisar ou criar a lista de tarefas críticas da unidade operacional. Pode-se escolher uma metodologia para definir as tarefas críticas ou utilizar as boas práticas para a decisão. Algumas tarefas, por sua natureza, já são consideradas críticas, por exemplo: partida e parada de plantas, falta de utilidades, cenários de perda de contenção, cenários críticos identificados em análise de risco mesmo que tenham camadas independentes de proteção, etc. 


\subsection{Relacionar os passos da tarefa e analisá-los com foco nos fatores humanos}

Definir os passos da tarefa com base na execução do trabalho ou em experiência do grupo de análise. Uma vez definidos os passos, analisar as possíveis interações humana-máquina e os seus respectivos potenciais de erro, quer seja de omissão ou de execução. Nesse momento devem ser considerados os fatores de modelagem de desempenho, a fim de analisar se há interferência em algum passo da tarefa e aumentar a possibilidade de erro. Alguns PSFs estão relacionados nas Tabelas 2 e 3.

Tabela 2 - PSFs externos

\begin{tabular}{|c|c|}
\hline S EXTERNOS & \\
\hline Características situacionais & Características da tarefa e equipamento \\
\hline 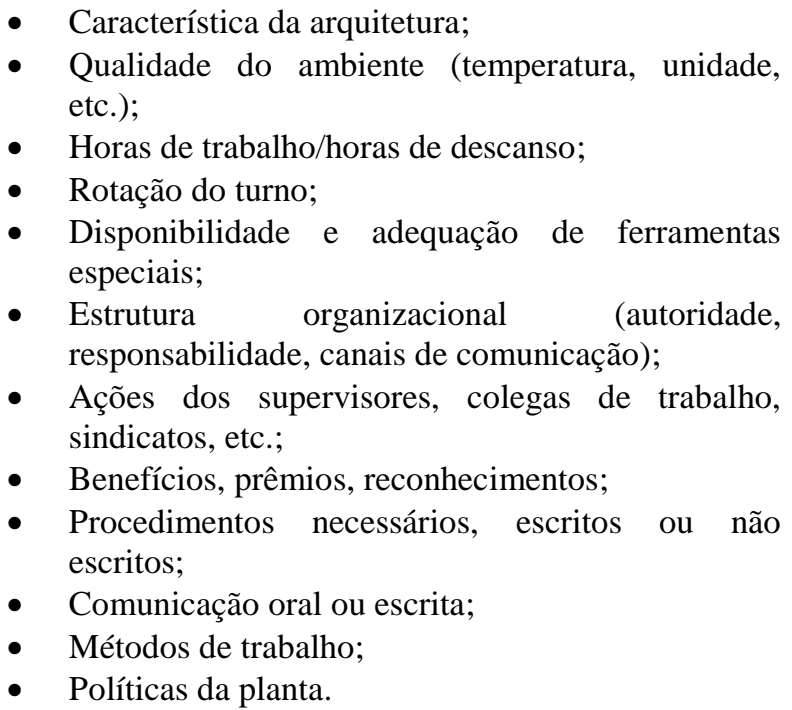 & $\begin{array}{l}\text { - } \text { Interpretação; } \\
\text { - } \quad \text { Decisão; } \\
\text { - } \quad \text { Frequência e repetitividade; } \\
\text { - } \quad \text { Criticidade da tarefa; } \\
\text { - } \quad \text { Memória curta e longa; } \\
\text { - } \quad \text { Cálculos necessários; } \\
\text { - Feedback (conhecimento dos resultados); } \\
\text { - } \quad \text { Dinâmica versus passo a passo da } \\
\text { - } \quad \text { atividade; } \\
\text { - Fatrutura do grupo e comunicação; } \\
\text { (projeto, teste de equipamento, fabricação } \\
\text { do equipamento, ferramentas, etc.). }\end{array}$ \\
\hline
\end{tabular}

Fonte: Adaptado de Swain e Guttmann (1983)

Tabela 3 - PSFs estressores e internos

\begin{tabular}{|c|c|}
\hline PSFs ESTRESSORES & PSFs INTERNOS \\
\hline $\begin{array}{l}\text { Estressores Psicológicos - Que afetam diretamente o } \\
\text { estresse mental }\end{array}$ & $\begin{array}{l}\text { Fatores pessoais - característica da pessoa } \\
\text { resultante das influências internas e externas }\end{array}$ \\
\hline $\begin{array}{l}\text { - } \quad \text { Rapidez inicial; } \\
\text { - } \quad \text { Duração do estresse; } \\
\text { - } \quad \text { Carga da tarefa; } \\
\text { - } \quad \text { Alto risco; } \\
\text { - } \quad \text { Ameaças (de falhas, do emprego, de perdas); } \\
\text { - } \quad \text { Período longo ou de baixa vigilância; } \\
\text { - } \quad \text { Conflito a respeito do desempenho do trabalho; } \\
\text { - } \text { nusência de reforço positivo e presença de reforço } \\
\text { - Distração (barulho, movimento, cor). }\end{array}$ & $\begin{array}{l}\text { - } \quad \text { Treinamento/experiência anteriores; } \\
\text { - } \quad \text { Estado da prática e habilidade atual; } \\
\text { - } \quad \text { Personalidade e inteligência variáveis; } \\
\text { - } \quad \text { Motivação e atitude; } \\
\text { - Estado emocional; } \\
\text { - Estresse (mental ou tensão no corpo); } \\
\text { - } \quad \text { Conhecimento dos padrões de } \\
\text { - desempenhos requeridos; } \\
\text { - } \quad \text { Conferença de sexo; } \\
\text { - } \quad \text { Atitude baseada na influência de família } \\
\text { ou outras pessoas. }\end{array}$ \\
\hline $\begin{array}{l}\text { ESTRESSORES PSICOLÓGICOS - PSFs que afetam } \\
\text { diretamente o estresse físico } \\
\text { - } \\
\text { - } \quad \text { Duração do estresse; } \\
\text { - } \quad \text { Fodiga; } \\
\text { - } \quad \text { Fome ou desconforto; }\end{array}$ & \\
\hline
\end{tabular}


- Temperatura extrema;

- Radiação;

- Força gravitacional extrema;

- Pressão atmosférica extrema;

- Insuficiência de oxigênio;

- Vibração;

- Limitação de movimento;

- Falta de exercício físico;

- Distúrbio do ritmo circadiano.

Fonte: Adaptada de Swain e Guttmann (1983)

Com o propósito de reduzir a probabilidade do erro humano, serão adicionados controles. Esses controles variarão conforme os comportamentos e tipos de erro. Erros de comportamentos baseados na habilidade são os deslizes/lapsos, e para minimizá-los os controles mais adequados são: 1) treinamento para melhorar a habilidade física e manipulativa por meio de práticas repetidas e feedback; 2) reforço nos procedimentos por meio de check list para ser verificado no início ou no fim da atividade; 3 ) melhoramento do layout, sinalizações e identificações de válvulas, tubulações e equipamentos. A falha de comunicação também pode gerar erro quando envolve habilidade. Daí a necessidade de melhorá-la, principalmente, a comunicação entre operador de painel e campo. Por exemplo, o operador de painel solicita ao operador de campo para fechar a "válvula X", então o operador de campo repetirá "fechar válvula X". Dessa forma, evitará a falha de comunicação no entendimento errado da ação a ser tomada, pois, ocorrerá redundância na comunicação.

Erros de comportamento baseados em regras estão relacionados com os enganos. Procedimentos errados ou complexos são normalmente as causas desses erros. Então, surge a necessidade da elaboração de procedimentos corretos e completos baseados em análise de tarefa, assim como, quando possível, fazer a simplificação da tarefa. Check lists também são úteis para evitar erros de omissão, principalmente se a tarefa é complexa, feita de forma não rotineira ou possui um elevado perigo intrínseco. Poderá ser preenchido antes ou após a realização da tarefa. Quando preenchido após, ajudará na recuperação do erro, no entanto, deve-se ficar atento ao tempo da segurança de processo que é aquele entre o evento iniciador e a consequência (CCPS, 2007). Se esse tempo for reduzido, é pouco provável que o check list seja eficaz. Nessa situação, treinamento e simulado exaustivo serão ações mais eficazes, como também o conhecimento detalhado do processo. Outro fator importante é a elaboração de uma planilha de solução de problemas a qual ajudará o operador a tomar decisões corretas. Finalmente, é fundamental o treinamento no procedimento e na planilha de solução de problemas.

Por fim, há os erros de comportamento baseados em conhecimento que também se relacionam com os enganos. Contudo, para evitar esse tipo de erro é preciso um investimento maior no armazenamento do conhecimento. Além dos procedimentos, será preciso empreender simulações para eventos complexos com intuito de assimilar das ações que devem ser tomadas. Além disso, o conhecimento mais profundo na tecnologia do processo, tais como, informações dos equipamentos, 
variáveis do processo e energias envolvidas, ajudará na tomada de decisão, mesmo se o procedimento não contiver todas as ações necessárias.

A redundância humana será outro recurso para situações críticas (SWAIN; GUTTMANN, 1983; API 770, 2001) nos casos de erros de comportamento baseados na habilidade e em regras. O grupo de análise de tarefa fará essa avaliação, todavia, considerará custos versus riscos aceitáveis, além de comparar com outras opções. Também, será fundamental avaliar se essa proposta é exequível no contexto organizacional.

\subsection{Elaborar procedimento}

O procedimento será o produto final da análise de tarefa. Se a análise for bem realizada, o procedimento terá consistência o suficiente para reduzir a probabilidade de erro humano. No entanto, o procedimento precisará ser elaborado de maneira tal que reduza possibilidade de erro de omissão ou execução. Os procedimentos serão escritos de forma clara e objetiva. Deve-se garantir que cada ação esteja separada por item para evitar erro de omissão. Se houver sucessivos equipamentos a serem alinhados, ficará claro no procedimento a identificação de cada um deles e a ordem de alinhamento. As ações estarão separadas de observações ou avisos, assim como evitar textos muito longos para facilitar o entendimento (CCPS, 1996; BRIDGES; WILLIAMS, 1997). Em adição, os procedimentos para tarefas críticas poderão ser suportados por check list em todos os passos ou apenas em passos que tenham a possibilidade de ocorrer um acidente. Ficarão disponíveis e com fácil acesso para serem consultados quando necessário.

\subsection{Treinamentos, simulados e oportunidades de melhoria}

O treinamento é um dos passos para tornar o procedimento conhecido por todos os operadores. Importante garantir a avaliação de retenção do conhecimento. Quanto aos simulados, podem ser por meio de simulação da tarefa pelo operador, acompanhado pela sua supervisão, ou ainda melhor, por pessoa que não trabalhe na mesma área para favorecer a independência. A pessoa que acompanhar o operador pode fazer perguntas ao longo do simulado, utilizando técnica de discussão sobre o simulado ou perguntas "o que acontece se” (SWAIN; GUTTMAN, 1983). A segunda opção é a simulação do cenário de acidente caso a realização da tarefa falhe. Outra maneira é selecionar algumas perguntas referentes ao procedimento e dirigir ao operador a ser avaliado. Por fim, uma alternativa adicional mais sofisticada para realizar o simulado da tarefa é por meio de simulador por computador, onde será possível simular as condições operacionais (CCPS, 1994). Ainda Swain e Guttmann (1983) sugerem que os simulados para tarefas mais críticas sejam realizados por mais de uma vez por ano, a fim de manter um aprendizado contínuo. A Figura 3 mostra um hipotético desempenho de emergência em função da realização de simulados. Percebe-se que quanto maior o tempo, muito menor será a eficácia de uma resposta à emergência se não houver 
simulados continuados. As dúvidas e questões do treinamento, resultados dos simulados e sugestões contínuas contribuirão para o aperfeiçoamento do procedimento.

Figura 3 - Efeitos hipotéticos da prática e não prática para manter a habilidade em emergências
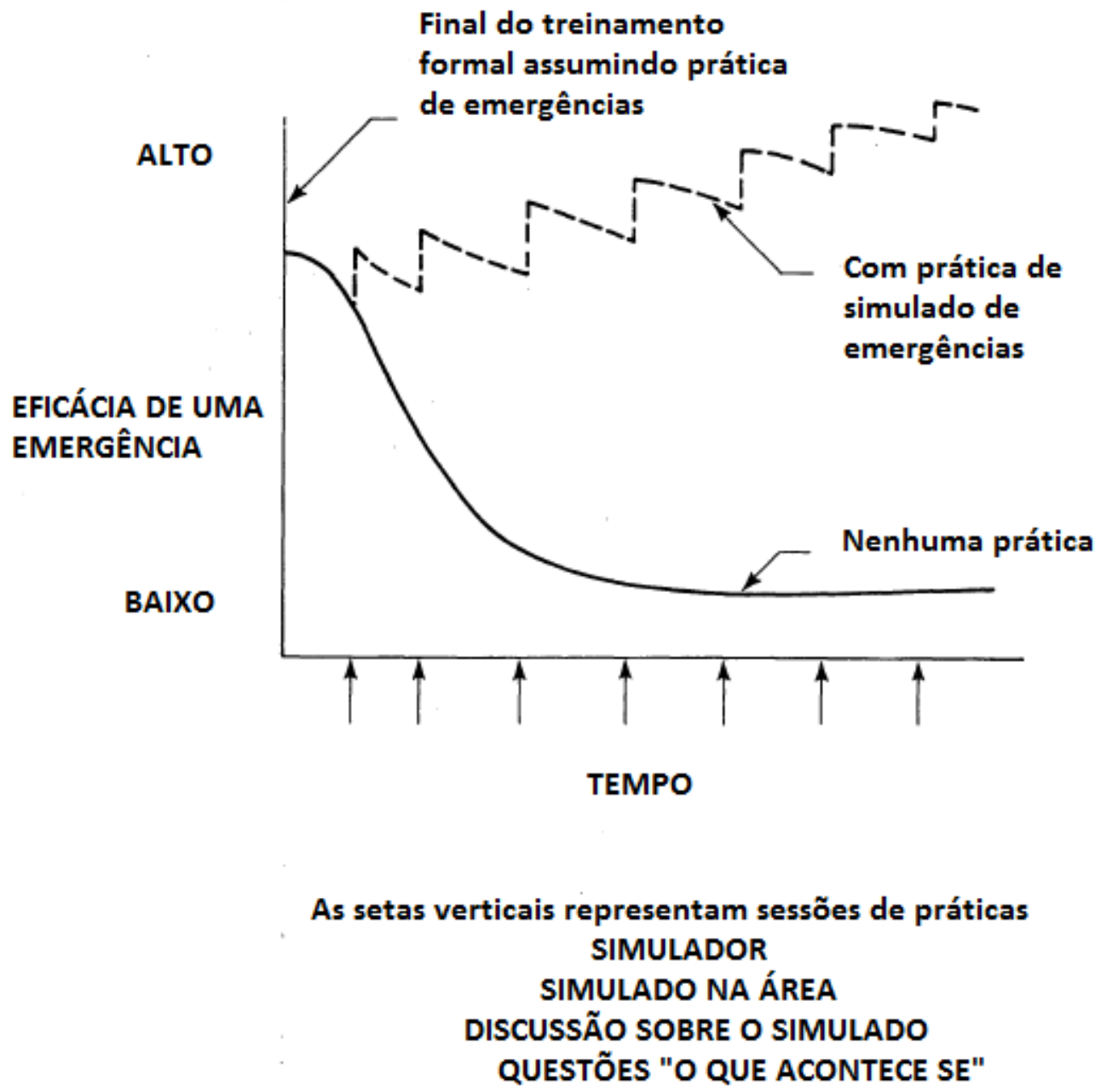

Fonte: Swain e Guttmann (1983)

\section{Erros por violações}

As violações não são incomuns. Às vezes, por uma pressão de produção um operador, ou até mesmo uma liderança, decide violar um procedimento para manter a planta operando. Se a violação for ordenada pela liderança, dará um sinal para os operadores que nem sempre deve ser seguido o procedimento da tarefa e em outras oportunidades as violações se repetirão. Para evitar essas situações, será preciso que a organização possua Disciplina Operacional (DO). A DO representa os princípios, atitudes e valores da organização e dos indivíduos que influenciam diretamente na segurança, qualidade e eficácia das operações (SILVA, 2009). Sendo assim, ao garantir que os elementos essências da DO estejam implantados na organização, as violações reduzirão.

A DO valorizará os comportamento alinhados à política da organização e enfatizará os seguintes fatores que modelarão o comportamento de todos: liderança atuante e definição de responsabilidade; determinação de objetivos e metas; todos honram os compromissos que foram 
discutidos e acertados; existência de documentações e procedimentos atualizados; intolerância a atalhos em procedimentos e regras; disponibilização de recursos suficientes e são utilizados de forma eficiente; reconhecimento das ações positivas por equipe ou individuais; há uma comunicação eficaz; existência de forte trabalho em equipe e envolvimento dos operadores; garantia do processo de melhoria contínua (SILVA, 2009).

\title{
8. Considerações finais
}

Erros humanos são causas de grandes acidentes no segmento industrial. Este artigo fez uma discussão dos erros humanos e tarefas. Destacou como os erros humanos interferem na segurança dos processos industriais e fez uma sugestão da gestão das tarefas. Redução de acidente no setor industrial passa por uma forte gestão dos erros humanos na execução da tarefa, principalmente aquelas consideradas críticas. Daí a necessidade de intensificar esforços na gestão das tarefas por meio de um processo que identifique as tarefas críticas, elabore os passos da tarefa, analise cada passo com o foco nos fatores humanos, elabore procedimento baseado nas informações obtidas na análise da tarefa, proporcione treinamento, simulados e avalie as oportunidades de melhoramento contínuo. Contra erros por violação o melhor remédio é a disciplina operacional disseminada em toda organização. Dessa forma os erros humanos reduzirão e os processos industriais se tornarão mais seguros.

\begin{abstract}
The human errors have been contributor factors of major accidents have already occurred at chemical, petrochemical, oil and gas industries. This occurs because automation in the industrial processes has increased due to technology development and the huge demands of consume. This phenomenon contributes to enhance the possibility of human errors, once the operators reduce the actuation mode from control to supervisory. Thus, they lose the ability to intervene in the process. Hence, with these facts arises the importance of special attention in tasks. The critical tasks have fundamental roles in the composition of the causes of accidents. Then, it is essential to improve the tasks management in order to reduce the probability of human errors. This paper seeks the better understanding of human errors by discussion of several authors and, finally, shows a model of tasks management that include identification of critical tasks, analysis of task steps, elaboration of procedure, training, drill e evaluation of improvement opportunity. The final outcome is the reduction of human errors probability regards critical task and hence the reduction of accident at industrial sector.
\end{abstract}

Key-words: tasks; management; accidents; human errors.

\section{Referências}

AMERICAN PETROLEUM INSTITUTE (API). A Manager's Guide to reducing Human Errors: Improving Human Performance in the Process Industries. Washington, DC: API, 2001.

ÁVILA, S. F.; PESSOA, F. L. P.; ANDRADE, J. C. S. Social HAZOP at an Oil Refinery. Process Safety Progress, New York: Vol.32, No.1, March, 2013. 
BELL, B. J.; SWAIN, A. D. A Procedure for Conducting a Human Reliability Analysis for Nuclear Power Plants. Albuquerque: Sandia National Laboratories, 1983. cross ref

BUREAU D’ENQUÊTES ET D’ANALYSES POUR LA SÉCURITÉ DE L’AVIATION CIVILE (BEA). Final Report of Accident with flight AF 447 Rio de Janeiro - Paris. Julho de 2012. Disponível em: < http://www.bea.aero/docspa/2009/f-cp090601.en/pdf/f-cp090601.en.pdf>. Acesso em: 15 jan. 2013.

BIRD, F. E.; GERMAIN, G. L.; CLARK, M. D.. Loss Control Management. 3th Duluth: Det Norske Veritas, 2007.

BRIDGES, W. LOPA and Human Reliability - Human Errors and Human IPLs. Março de 2010. Disponível em: <http://www.piii.com/_downloads/LOPA_and_Human_Factors_2.pdf>. Acesso em: 10 mai. 2012.

BRIDGES, W. G.; WILLIAMS, T. R. Create Effective Safety Procedures and Operating Manuals. Chemical Engineering Progress, New York, dec. 1997.

CENTER FOR CHEMICAL PROCESS SAFETY. Guidelines for Preventing Human Error in Process Safety. New York: American Institute of Chemical Engineers, 1994.

CENTER FOR CHEMICAL PROCESS SAFETY. Guidelines for Safe and Reliable Instrumented Protective Systems. New Jersey: John Wiley \& Sons, 2007.

CENTER FOR CHEMICAL PROCESS SAFETY. Writing Effective Operating and Maintenance Procedures. New York: American Institute of Chemical Engineers, 1996

EMBREY, D.; ZAED, S. a Set of Computer Based Tools Identifying and Preventing, Human Error in Plant Operations. 2006. Disponível em: <

http://www.humanreliability.com/articles/Computer\%20based\%20tools\%20website\%20version.pdf>. Acesso em: 05 mai. 2013.

GELLER, E. S. The Psychology, of Safety, Handbook. Boca Raton: CRC Press, 2000. crossref

HEALTH AND SAFETY EXECUTIVE. Human factors in the management of major accident hazards. Outubro de 2005. Disponível em: <http://www.hse.gov.uk/humanfactors/topics/toolkitintro.pdf>. Acesso em: 20 nov. 2010.

HOLLnAGEL, E.; WOODS, D. D.; LEVESON, N. Resilience Engineering: Concepts and Precepts. Burlington: ASHGATE, 2008.

LEVESON N. G. Engineering a Safer World: Systems Thinking Applied to Safety. Cambridge e Massachusetts: Massachusetts Institute of Technology, 2011.

LIDWELL, W; HOLDEN, K; BUTLER, J. Universal Principles of Design: 125 Ways to Enhance Usability, Influence Perception, Increase Appeal, Make Better Design Decisions, and teach Through Design. Massachusetts: Kindle Edition, 2010.

MANNAN, S. Lee's Loss Prevention in the Process Industries. $3^{\text {rd }}$ Edition. Burlington: Elsevier, 2005.

MARINE BOARD. Macondo Well-Deepwater Horizon Blowout: Lessons for Improving Offshore Drilling Safety. Washington: National Academy of Engineering and National Research Council, 2011.

PERROW, C. Normal Accidents: Living with High-Risk Technologies. United States of America: BasicBooks, 1984.

RASMUSSEM, J. Human Errors. A Taxonomy for Describing Human malfunction in Industrial Installations. Journal of Occupationai Accidents, 4 (1982) 311-333. cross ref

RASMUSSEM, J. Skills, Rules, and Knowledge; Signals, Signs, and Symbols, and Other Distinctions in Human Performance Models. IEEE Transactions on Systems, Man, and Cybernetics, v. SMC-13, n. 3, 257-266, May/June 1983. cross ref

REASON, J. Human Error. United Kingdom: Cambridge University Press, 1990. crossref

REED, J.; SHAFFER, M. The Incorporation of Human Factors Engineering into the Design of the AP1000 Nuclear Power Plant. In: Power Industry Division Symposium, 2008. Scottsdale, Arizona. Anais eletrônicos... International Society Automation. 
SILVA, E. C. Por Que Alguns Programas de Segurança São Ineficazes? In: XXIX Encontro Nacional de Engenharia de Produção. 2009, Salvador. Anais... Salvador: ENEGEP, 2009. p. 1-8.

SWAIN, A. D.; GUTTMANN, H. E. Handbook of Human Reliability Analysis with Emphasis on Nuclear Power Plant Applications - Final Report. Albuquerque: Sandia National Laboratories, 1983.

\section{Dados do autor}

Nome: Elísio Carvalho Silva

Instituição: Universidade Federal da Bahia - UFBA - Bahia - Brasil

Cargo: Estudante do Mestrado de Engenharia Industrial

Endereço: Praça, Igaratinga, 206, Condomínio Monte Belo, Edf. Vista Alegre, Apto 801. Cep41830290.

Telefone: (71) 92226466

e-mail: elisio.carvalho@terra.com.br

Submetido em: 02/07/2013

Aceito em: 08/09/2014 